\title{
Design of Port Unattended Weighbridge System Based on Internet of Things
}

\author{
Weikeng Lin ${ }^{1,}$, Lei Huang ${ }^{2, b}$ \\ ${ }^{1}$ School of Economics and Management, Beijing Jiaotong University, Beijing 100044, China \\ ${ }^{2}$ School of Economics and Management, Beijing Jiaotong University, Beijing 100044, China \\ a16120609@bjtu.edu.cn, blhuang@ bjtu.edu.cn
}

\begin{abstract}
Trucks are one of the most important tools for port transportation. However, at present, many ports companies in China have a lower level of intelligence, even some port companies still use the on-site guidance methods of weighman to weigh. This kind of manual intervention has low efficiency, high error rate, high labor cost and high risk of cargo. Therefore, designing an efficient weighbridge system is especially important for port companies to improve the management of weighing. Based on Internet of Things technology, this paper designed an unattended weighbridge system. The unattended weighbridge system combines the Internet of Things weighbridge with a production business system. The Internet of things weighbridge is responsible for collecting weighing data while the production business system is responsible for processing data and sending commands. This system has achieved the automation of weighing, improved the efficiency of weighing, saved manpower cost, reduced the risk of cargo, which has high practical value.
\end{abstract}

Keywords: port, weighing, Internet of things, unattended weighbridge system.

\section{Introduction}

The process of weighing is a very important part of the port transportation, and the efficiency of the weighing is of great significance to the service quality and economic benefit of port enterprises. Throughout the domestic port enterprises, some port enterprises still use the on-site guidance of weighmen to weigh. This kind of manual intervention needs weighmen to work shift, transfer tickets to drivers face to face, which has low efficiency, high error rate, high labor cost and high risk of cargo. Therefore, it is very important for port to adopt new technology to improve the management mode of weighing.

As one of the new generation of information technology, the Internet of things has been used by more and more industries because of its ability to connect objects to the Internet. With the help of Internet of things technology, port enterprises can realize the automation of truck weighing process to achieve the goal of saving manpower, improving efficiency and reducing risks of goods. At present, many domestic and foreign scholars have studied the use of Internet of things technology. Zeng Fei of Wuhan University of Technology has designed a port weighbridge management system based on license plate recognition ${ }^{[1]}$. The system uses image recognition to identify the license plate number and upload it to the control center for automatic weighing. There are some defects in the scheme: First, the distance of license plate recognition is relatively short, and the recognition rate is greatly reduced in bad weather. Second, the method based on the license plate recognition cannot effectively monitor the cheating behaviors, and there is a large risk of cargo. China Utility Model Patent No. 202562616U discloses a remote weighbridge data transmission system based on the Internet of Things ${ }^{[2]}$. The system uses a non-contact IC card data acquisition module to connect to a monitoring center, and the control center controls weighing. However, the design scheme has the following defects: First, the read-write distance between the non-contact IC card and the IC card reader is short, only $5-10 \mathrm{~cm}$; Second, the on-site controller and the monitoring center failed to achieve centralized control, and the anti-cheating method was low. 
In view of these circumstances, this paper proposes a port unattended weighbridge system based on the Internet of Things. The system uses an RFID tag as a vehicle identifier. With the aid of sensors, cameras, and other equipment, it can realize the automation of weighing in order to save manpower cost, improve efficiency, reduce cargo risk, and achieve the goal of data sharing.

\section{Introduction of related technology}

\subsection{Introduction of The Internet of things}

The typical Internet of things consists of RFID systems, middleware Savant systems and Internet systems $^{[3]}$, as shown in figure 1 . RFID system mainly includes electronic tag, reader and data exchange and management system software; The middleware Savant system consists of Savant server, ONS server, PML server and corresponding data software. Internet systems are usually composed of computer systems and network servers.

In the IoT system shown in figure 1, after receiving the carrier information from the electronic tag and demodulating and decoding the received signal, the reader transmits the information to the computer middleware Savant system for processing. The Savant system transmits that processed information to the Internet and then use ONS to find the stored location on the Internet. The ONS indicates to the Savant system that the item-related information server is stored, and transmits the information on this item in the document.

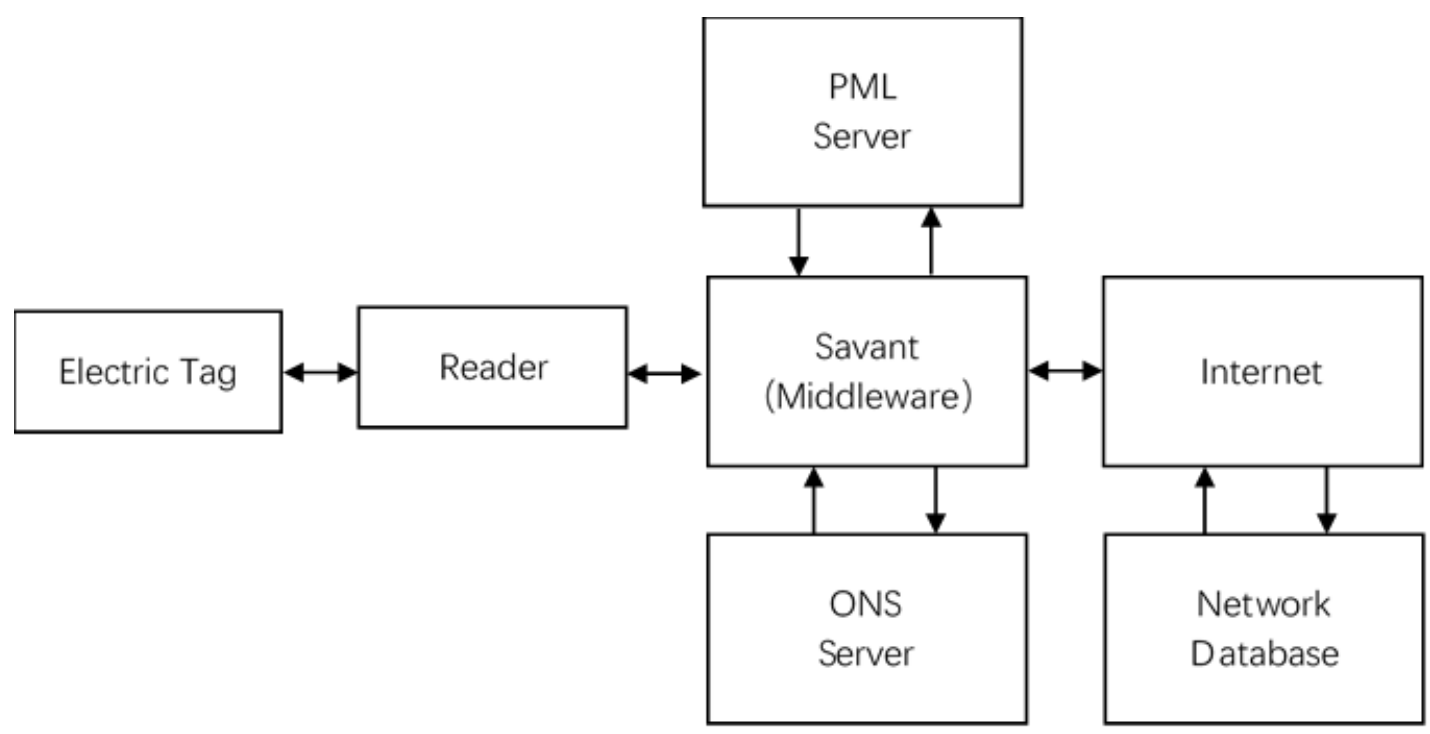

Fig. 1 The framework of the Internet of things

\subsection{Introduction of the Socket communication}

Two programs on the network exchange data through a two-way communication connection, one end of this connection is called a socket.

Depending on the way the connection is started and the target of the local socket to connect to, the connection process between sockets can be divided into three steps: server listening, client requests, connection confirmation $^{[4]}$.

1) Server monitoring: It is the server-side socket that does not locate the specific client socket but is waiting for the connection and monitors the network status in real time.

2) Client request: refers to a connection request made by the client's socket. The target to be connected is the server's socket. To do this, the client's socket must first describe the socket of the server to which it is connected, indicate the address and port number of the server socket, and then make a connection request to the server socket.

3) Connection Confirmation: When a server-side socket listens to or receives a client socket connection request, it responds to the client socket request, creates a new thread, and sockets the server. The description of the word is sent to the client. Once the client confirms the description, the 
connection is established. The server-side socket continues to listen and continues to receive connection requests from other client sockets.

\section{The design of port unattended weighbridge system}

\subsection{The structure of unattended weighbridge}

This paper designs an unattended weighbridge system based on Internet of things, the hardware devices involved including weight sensor, ground loop, infrared sensors, RFID antenna, camera, LED display screen, printer, barrier gate, indicator light, and industrial control computer. Among them, the industrial control computer is the control center of the whole system, which functions as receiving data, transmitting data and sending instructions. The concrete structure of unattended weighbridge is shown in figure 2 .

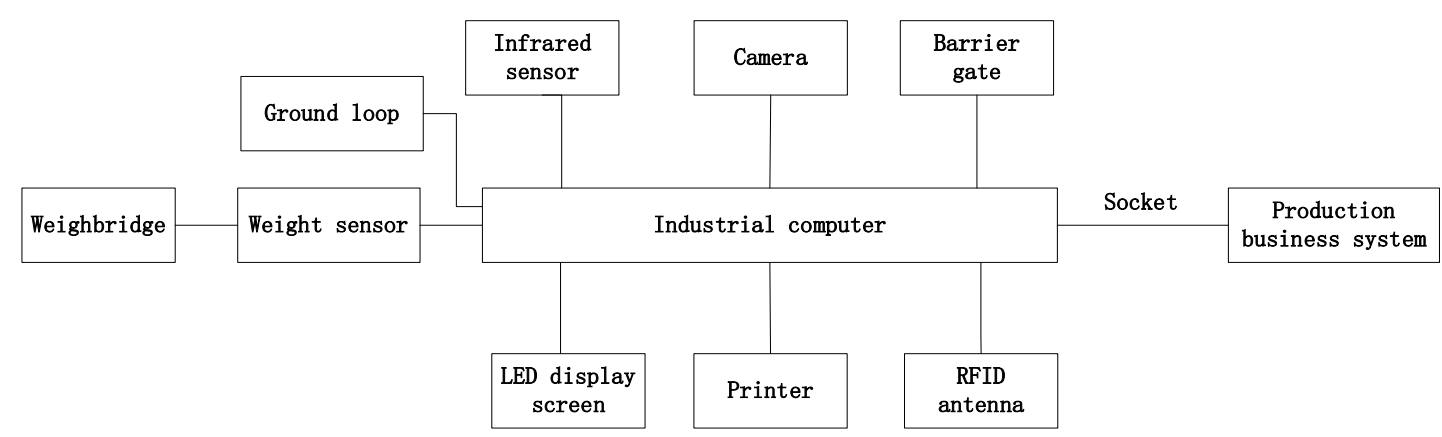

Fig. 2 The structure of the weighbridge system

The following is an introduction to the layout of unattended weighbridge and the functions of each hardware.

The weighbridge itself has a weight sensor. Infrared sensors and ground loops are provided on the front and rear ends of the weighbridge. Cameras are installed on the front, rear, side and top of the weighbridge. Barrier gate and indicator light are installed at the exit of the weighbridge. The side of the weighbridge is equipped with an RFID antenna, a printer, and an LED display. Inside the weighbridge room is an industrial computer.

The industrial control computer is connected with the weight sensor, infrared sensor, ground loop, cameras, barrier gate, indicator light, RFID antenna, printer and LED display screen. Its functions include:

1) Receiving the data information of weight sensor, infrared sensor, ground loop, RFID antenna and cameras;

2) Through the socket communication, the data received in the production business system is transmitted to the production business system through the written interface;

3) Receiving the instruction information sent by the production business system and control the relevant actions of the printer, barrier gate, indicator light and LED display according to the instruction information.

The weight sensor is used to measure the steady weight of the truck and pass the measured weight to the industrial control computer.

The infrared sensor is used to detect the position of the truck. When the truck is fully loaded, the infrared sensor is completely closed and the system will begin to collect data. 
The ground loop is used to detect trucks moving in and out of the weighbridge. When the truck triggers the ground loop at the entrance of weighbridge, the RFID antenna will be started by the industrial control computer to read the RFID card information. When the truck triggers the ground loop at the exit, the barrier gate will be closing through the industrial control computer.

The cameras are used to capture images and videos, and pass it to the monitoring room. The pictures and videos will be stored in the storage medium for the purpose of searching.

The indicator light is used to receive the instruction sent by the industrial control computer to show the current state of barrier gate.

The barrier gate is used to receive the instructions sent by the industrial control computer and carry out the operation of lifting or falling.

The RFID antenna is used to identify RFID tags and transmit RFID card information to industrial control computer.

The printer is used to receive the instructions sent by the industrial control computer and print the pick-up ticket.

LED display screen is used to display the current status, weight and other information for the driver to understand the situation.

\subsection{The weighing process of unattended weighbridge}

The weighing process of unattended weighbridge is shown in Figure 3.

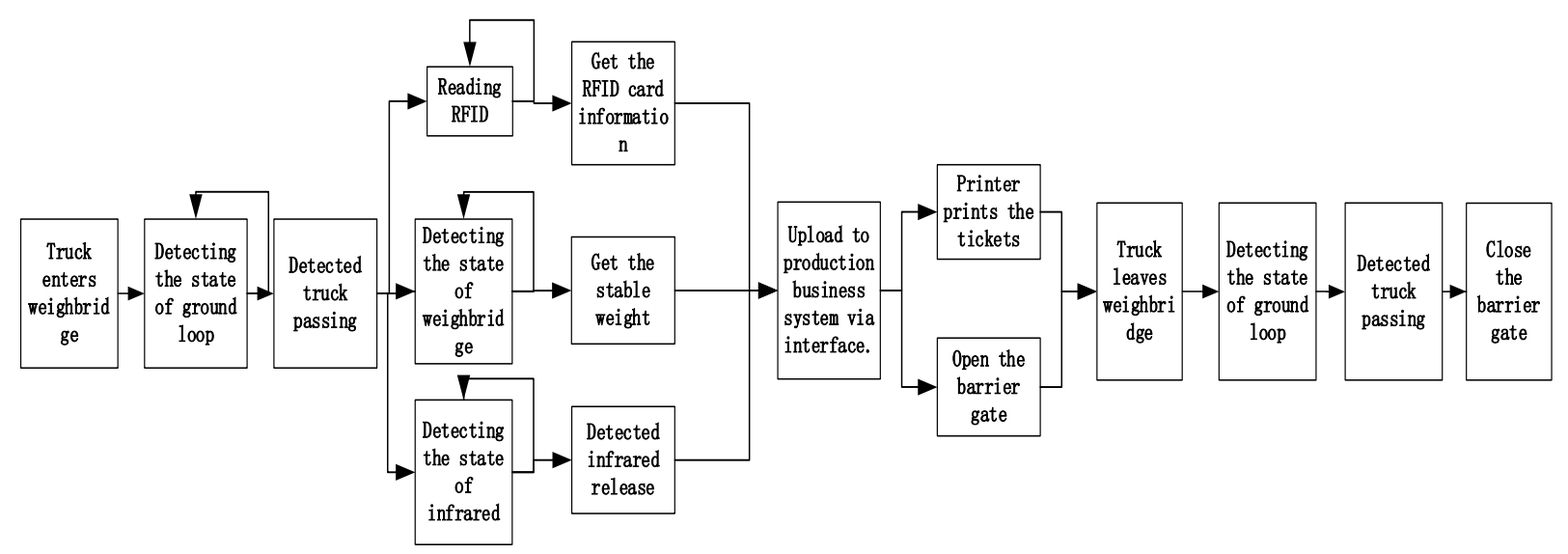

Fig. 3 The weighing process of unattended weighbridge

The complete weighing procedure is as follows:

1) When the truck arrives at the entrance of the weighbridge, it triggers the group loop at the entrance. At this time, the RFID reading and writing device is started and the RFID card information pasted on the windshield of the truck is read. After the truck fully enters the weighbridge, two pairs of infrared sensors at both ends of the weighbridge are fully closed. At this time, the weight sensor measures the truck's stable weight value.

2) The industrial control computer receives the RFID information obtained by the RFID antenna and the weight value obtained by the weight sensor, then transmits it to the production business system. The production business system reads the vehicle information and cargo information from the database through the RFID information, so as to carry out judgment rules.

3) The production business system determines whether the barrier gate is open according to the established rules for weighing:

If it is judged that the truck can be weighed, then save the weighing record to the database, and send the print information and the release information to the industrial control computer. The industrial control computer sends the relevant instructions to the printer and the barrier gate, and the printer automatically prints the delivery receipt. When the barrier gate is opened and the truck is 
completely driven out, it triggers the group loop at the exit of the weighbridge and the barrier gate closes.

If it is determined that the truck cannot be exceeded, the barrier gate will not be opened and the LED display will give the reason why the vehicle cannot weigh.

\section{Conclusions}

This paper combines the Internet of Things technology, designed an unattended weighbridge system for port companies. The unattended weighbridge system can realize automation of weighing, improve the efficiency of weighing, save manpower cost and reduce cargo risk, which has high practical value.

\section{References}

[1] Zeng Fei. Port weighbridge management system based on license plate recognition[D]. Wuhan University of Technology, 2007.

[2] Xu Jianhua, Hu Xianghua, Lu Yiliang, et al. Long-distance weighbridge data transmission system based on the Internet of Things: CN202562616U[P]. 2012.

[3] Li Runian. Research on Internet of Things based on RFID technology[J].Journal of China Academy of Electronics and Information Technology,2009,4(06):594-597.

[4] Ou Jun, Wu Qingxiu, Xu Yun, Zhang Hong.Research on Network Communication Technology Based on Socket[J].Net Security Technologies and Application,2011(07):19-21. 